\title{
Laparoscopic Approach of the Obstruction of the Lower Third of the Esophagus in Children
}

\author{
Carlos García-Hernández, MD, Lourdes Carvajal-Figueroa, MD, Cristian Archivaldo-García, MD, \\ Carlos Pique-Aragon, MD, and Sergio Landa-Juarez, MD
}

\begin{abstract}
Objective: To review the diagnostic methodology in pediatric patients with obstruction of the lower third of the esophagus as well as minimally invasive therapeutic options.

Materials and Methods: Retrospective study carried out reviewing records of children with esophageal obstruction diagnostic, from 2000 to 2018. They were divided into Group I stenosis secondary to reflux; Group II achalasia; and Group III embryonic remnants.

Results: Thirty-three patients. Group I: 7; esophageal barium swallow irregular stenosis of the distal third and endoscopy irregular stenosis in 7. Treated with laparoscopic fundoplication 2, Collis Nissen 5. Group II: 22 patients, age $X=11.55$ years. All with dysphagia and symmetrical stenosis of esophagogastric junction. Fifteen underwent manometry and all underwent intraoperative endoscopy. All had laparoscopic myotomy, with 2 perforations and no conversions, 2 patients had subsequent dysphagia to solids, and they did not need esophageal dilatation. Group III: 4 patients, stenosis was above esophagogastric junction. On endoscopy, inflammation was present in all 3 with irregular esophagogastric junction and difficulty passing endoscope. Three patients underwent laparoscopic resection and anastomosis. One patient leaked and developed a fistula. One patient has not been operated upon as yet.

Conclusions: In those patients, the best surgical option depends upon the diagnosis. Esophageal barium studies and endoscopy allow discerning among them.
\end{abstract}

Keywords: esophageal stenosis, gastroesophageal reflux, achalasia, embryonic remnants

\section{Introduction}

T HE NATURE OF DISEASES that can potentially harm the whole esophagus or specific portions of it is varied. ${ }^{1}$ For instance, causes rendering disruptions to regular food intake through the lower third of the esophagus could be intramural, of congenital origin, as is the case with embryonic remnants $^{2-4}$; acquired, via gastroesophageal reflux ${ }^{5-7}$; or even due to changes in esophageal motility, as seen in achalasia patients. ${ }^{8}$

Nevertheless, each of these conditions is quite rare. Embryonic remnants are estimated to be present in one out of 25,000-50,000 living newborns. Reflux associated with esophageal stenosis happens in $7 \%$ of patients with esophagitis. In fact, despite both of these conditions could potentially damage any segment of the esophagus, the lower third is consistently affected the most. ${ }^{3,9}$ Finally, the incidence of achalasia is a mere 1.1 out of a million children. ${ }^{10,11}$ Given the specific surgical treatments for these pathologies and the acute complications that can arise from failing to perform them accordingly, the importance of an accurate diagnosis cannot be overstated.

Against this backdrop, this study seeks to review the diagnostic methodology in pediatric patients with obstruction of the lower third of the esophagus, while recounting our experience with different minimally invasive alternatives.

\section{Materials and Methods}

Retrospective review of records for 33 patients aged 18 years and younger diagnosed with obstruction of the lower third of the esophagus. After performing paraclinical tests comprising barium esophageal swallow, upper digestive endoscopy, test of $\mathrm{pH}$-impedance, manometry, and assessing 


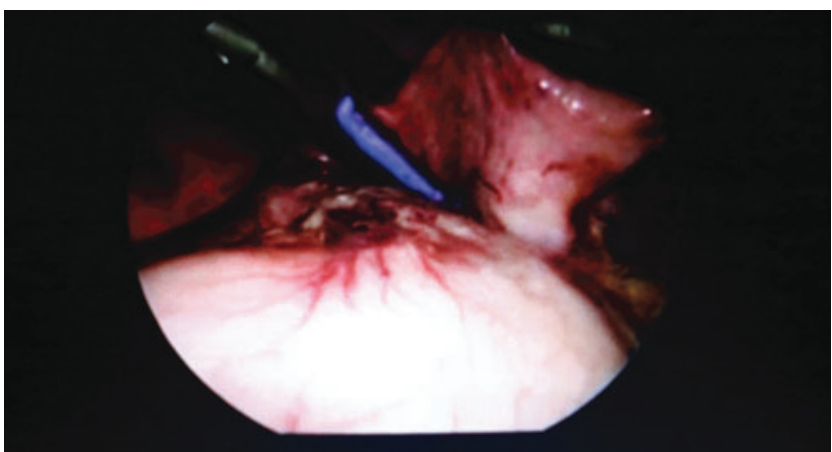

FIG. 1. Collis procedure in a patient with shortened esophagus and stenosis, gastroplasty is observed.

age, sex, symptomatology and previous treatments, the sample was divided into three groups as follows:

- Group I-Patients with stenosis of the lower third of the esophagus secondary to gastroesophageal reflux.

- Group II-Achalasia patients.

- Group III-Patients with congenital embryonic remnant obstruction.

Within Group I, $360^{\circ}$ Nissen fundoplication and four postoperative sessions of Savary esophageal dilation were conducted in patients with no esophageal shortening, while a Collis-Nissen laparoscopic procedure using a $45 \mathrm{~cm}$ linear stapler (Fig. 1) and 5 postoperative sessions of Savary esophageal dilation were performed on those with esophageal shortening. Individuals in Group II underwent a Heller esophageal myotomy with endoscopic control, comprising $4 \mathrm{~cm}$ of esophagus above junction and $2 \mathrm{~cm}$ below it in the anterior side of the stomach (Figs. 2 and 3) linked to a $180^{\circ}$ posterior partial fundoplication sutured to the edges of the myotomy. Finally, patients in Group III went through an esophageal resection of the affected segment with end-to-end anastomosis in a single plane with $2-0$ polyester suture, linked with a fundoplication (Fig. 4). In later sections of the work, we present our data and assess results for surgical time, intraoperative complications, conversion, oral intake tolerance, drug requirements, and follow-up time using descrip-

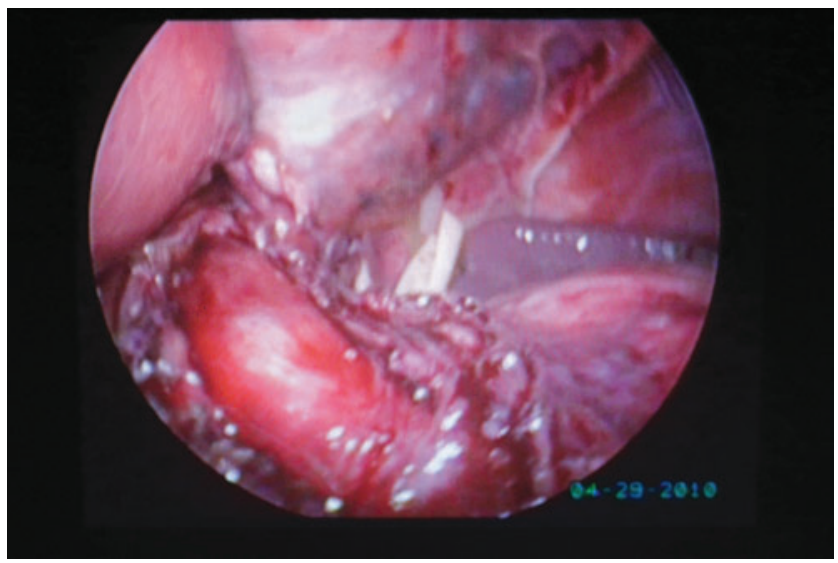

FIG. 2. Esophageal myotomy in a patient with achalasia, integrity of esophageal mucosa is seen.

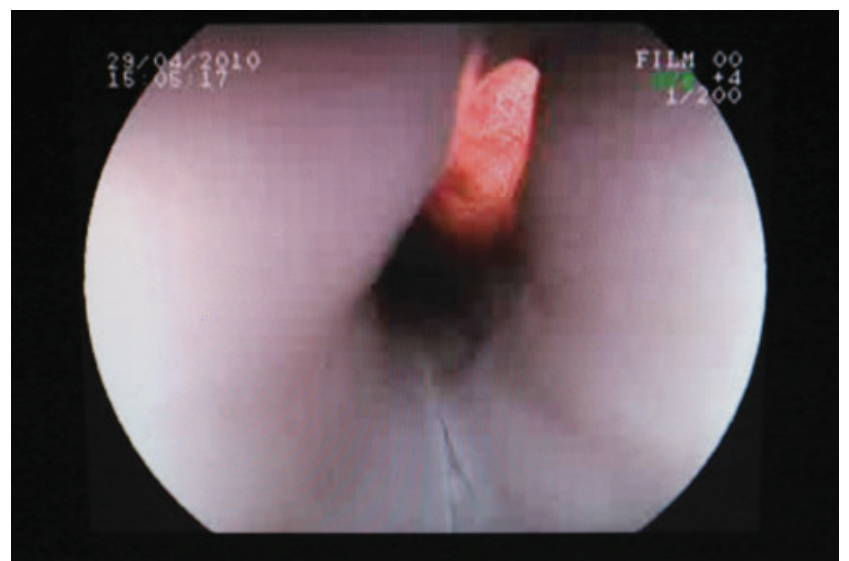

FIG. 3. Endoscopic image after Heller myotomy where its length could be appreciated.

tive statistics of these variables, particularly measures of relative and central frequency. Of note, the outcomes were reported by a researcher with no link to the procedures performed and consulted by us for the purpose of this study.

\section{Results}

A total of 33 patients were treated from 2000 to 2018.

Group I included 7 subjects, 4 males and 3 females, all with stenosis, 5 with short esophagus, and 2 with normal esophageal length. Ages ranged from 4 to 15 years with a mean of 6.83 years. All of them showed dysphagia, 5 out of 7 also presented pyrosis and cough, while the remaining 2 reported abdominal pain. Esophageal barium swallow revealed irregular stenosis with esophageal shortening in 5 children (Fig. 5a), while 2 individuals showed an irregular decrease in caliber without shortening. In addition, test of $\mathrm{pH}$-impedance revealed pathologic gastroesophageal reflux in all 7 patients. Endoscopies reported punctiform stenosis with esophagitis of the distal third and high gastroesophageal junction line (Fig. 6a). Laparoscopic $360^{\circ}$ Nissen fundoplications were performed in 2 patients and Collis-Nissen esophagoplasties were conducted in the 5 children who presented shortening.

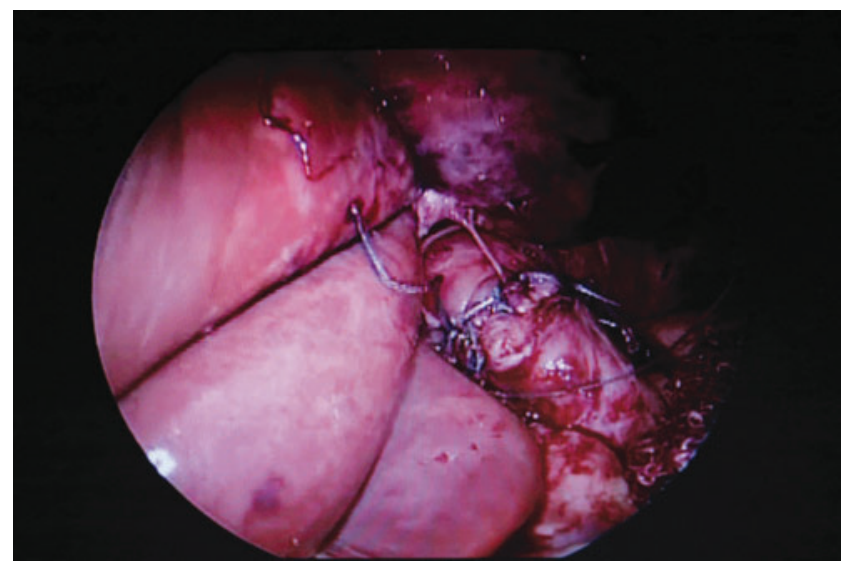

FIG. 4. End-to-end esophageal anastomosis after resection of injured segment. 


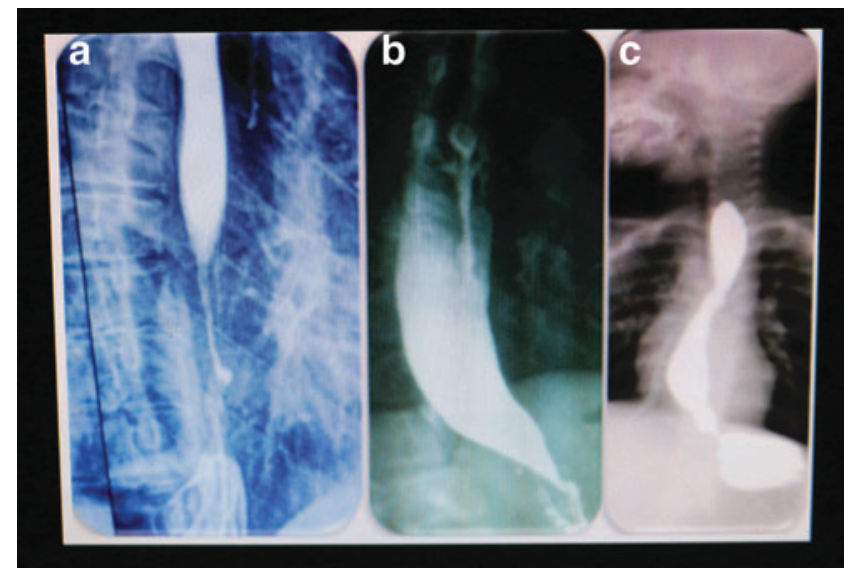

FIG. 5. Contrast studies: (a) stenosis due to reflux, (b) achalasia, (c) embryonic remnants.

Four sessions of postoperative Savary esophageal dilations were applied in all patients. No conversions or intraoperative complications arose. Surgical time ranged from 45 to 150 minutes, with a mean of 87.8 . Oral intake began 8 hours after the procedure in children with fundoplication and 3 days later in those with esophagoplasties. Total remission of symptoms was reported in 5 children, while 2 subjects received occasional antacids. Follow-up period spanned from 8 months to 10 years.

Group II comprised 22 individuals, aged from 1 to 20 years with a mean of 11.5, the youngest of which presented Allgrove syndrome (achalasia, alacrimia, and renal insufficiency). ${ }^{12}$ Symptom evolution was in the 4- to 36-month range, with a mean of 16.45 . Dysphagia was present in all patients within this group, with 11 showing regurgitation and retrosternal pain and 12 displaying cough and respiratory symptoms. In terms of treatment history, 17 patients had received antireflux treatment with prokinetics and antacids, 4 had been dilated, and 2 had gone through esophageal myotomy by thoracotomy procedures, with persistency of dysphagia in all cases. Esophageal gastroduodenal series revealed symmetrical and regular caliber decreases reaching esophageal junction across the board, while food debris was also present in some cases (Fig. 5b). Manometries were performed in 15 patients, rendering no relaxation of the inferior esophageal sphincter in 10 individuals and being inconclusive in one patient, whereas no such procedure was carried out in 7 patients due to equipment unavailability, and diagnosis was assessed by means of radiologic and endoscopic findings instead. The remaining 4 patients chose not to cooperate with this study. Endoscopies were performed in all 22 patients, showing dilated esophagus with saliva and images of concentric folds, no distension with air insufflation, and easy passage of endoscope and normal retrovision (Fig. 6b) in all cases. In addition, all patients underwent Heller miotomy procedures with intraoperative endoscopic control. Surgical time varied between 65 and 110 minutes, with a mean of 78.33 , with no conversions, two mucosal perforations occurred which were repaired within the reported surgical time. Occasional pyrosis in 4 patients, with infrequent dysphagia in 2 cases, warranted prescribing antacids. Favorable evolution was shown by 16 subjects with follow-up spans greater than or equal to 5 years. Meanwhile, within the 6 patients with $<5$ years of postoperative evolution, 2 displayed occasional dysphagia although no dilatation was needed in any case. Other 2 patients reported gastroesophageal reflux.

Group III encompassed 4 patients with congenital embryonic tracheobronchial remnants, in the 18- to 30-month age range, 3 females and 1 male. All had previously received antireflux medication, showed dysphagia, vomiting, and low weight. Besides, two reported cough and bronchospasm. Esophagus barium swallow showed dilation in the lower third of the esophagus, above the esophageal junction with a small normal distal portion of the esophagus (Fig. 5c). Manometries were executed in two infants displaying absent relaxation of the lower esophageal sphincter. Endoscopies were performed in all patients, revealing eccentric and irregular stenosis of the lower third of the esophagus, without affecting the esophageal junction, with normal retrovision (Fig. 6c). Resection of injured segments was conducted in 3 patients, showing whitish spots on the esophageal surface, with small normal distal segment from esophagus and junctions (Fig. 7a), histopathological report showed cartilage remnants in these three cases (Fig. 7b). Surgical times were 2.3, 2.5, and 3.1 hours, with 1 patient awaiting surgery at the time of writing. One patient presented an anastomotic leak on the fifth postoperative day and, thus, required a new operation and extension of the gastrostomy feeding period. The remaining 2 infants proceeded to feed orally on the fifth day after surgery.

\section{Discussion}

Lower third esophageal obstruction can stem from a variety of rare pathologies. ${ }^{3,5,10,11,13,14}$ Even when the esophagus might have several congenital lesions, this 33-case survey showed these could arise from gastroesophageal reflux, achalasia, or cartilaginous embryonic remnants. Even

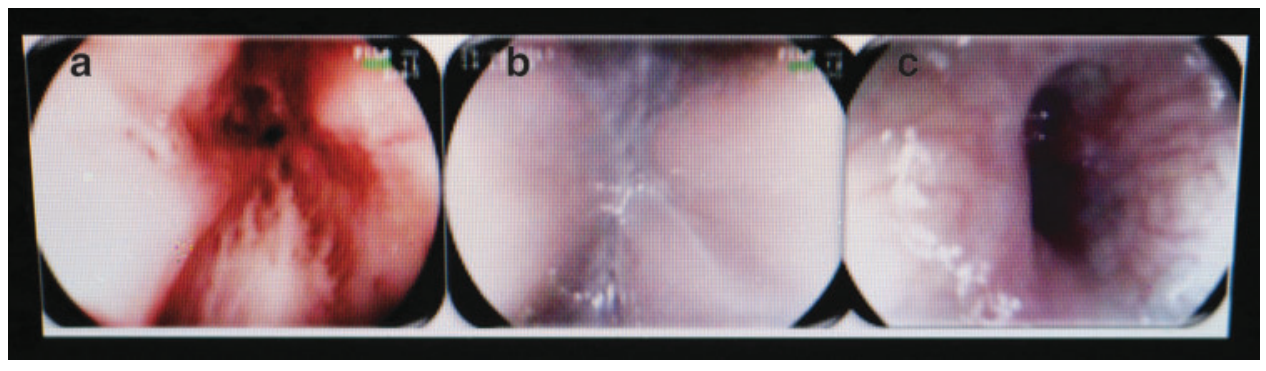

FIG. 6. Endoscopic images. (a) Stenosis due to reflux, (b) achalasia, (c) embryonic remnants. 

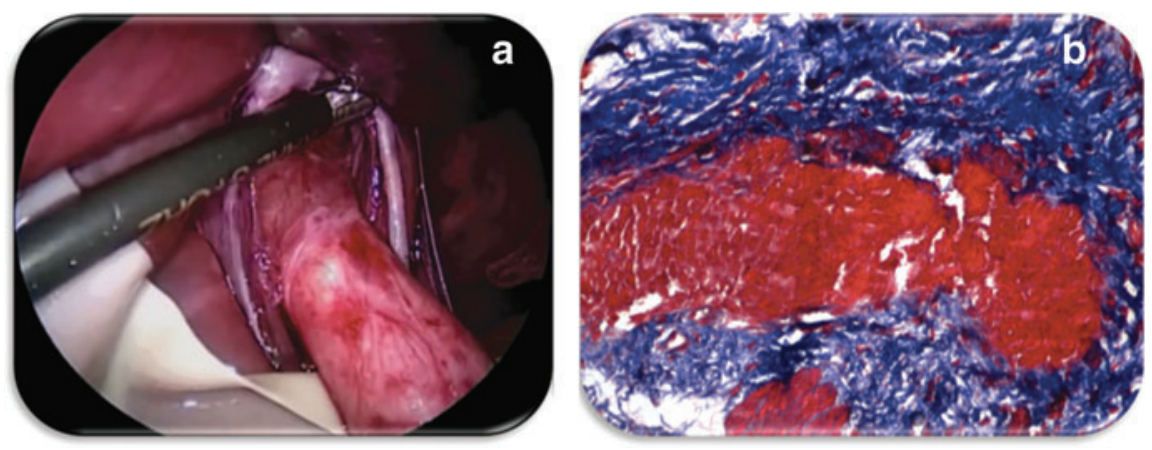

FIG. 7. (a) Intraoperative image, injured esophagus. (b) Histopathological study with cartilaginous remnants.

though symptomatology is similar in all three cases, a proper assessment at the beginning of condition could appropriately direct diagnosis. Age also provides a valuable lead, although it should not be taken as a conclusive indicator by itself as shown by this study. Younger patients in this series tended to present embryonic remnants and individuals with reflux or achalasia were on average older, nonetheless, a year-old infant who reported a syndromic modality of achalasia (Allgrove's syndrome ${ }^{12}$ ) was also recorded.

Paraclinical studies are very useful for diagnosis; esophagram series allow for discrimination among reflux-related lesions, embryonic remnants, or achalasia, but undoubtedly, endoscopy carries the most weight in establishing a final diagnosis in this kind of patients. Stenosis due to reflux could occur anywhere in the esophagus, but incidence is most common in the lower third, ${ }^{5,6}$ its effect could reach the esophageal junction, with inflammatory data in the lower third with a friable mucosa, ${ }^{9}$ as found in patients surveyed in this work. Endoscopies performed in patients with achalasia revealed no anatomical obstruction as the endoscope could go through toward the stomach, ${ }^{11}$ with normal esophageal mucosa and a lack of cardia opening with air insufflation. In contrast, for children with embryonic remnants, endoscopy revealed irregular mechanical obstruction above the esophageal junction, as described previously. ${ }^{4}$

Abnormal $\mathrm{pH}$ impedanciometry in patients with reflux improved the diagnosis. In contrast, the usefulness of manometries for refining diagnosis in children with achalasia proved limited for three main reasons; equipment unavailability, a requirement of patients' cooperation, and the difficulty of performing the study itself. Moreover, mechanical obstruction or pseudoachalasia could be mistaken for achalasia, since impaired relaxation of the lower esophageal sphincter accompanied by lack of peristalsis of the esophageal body could be present. ${ }^{11,15}$ In fact, this was observed in two patients with embryonic remnants included in this survey; although manometries reported achalasia, endoscopic procedures pointed to mechanical obstruction in both cases, which was corroborated through histopathological studies.

As mentioned earlier, the importance of diagnostic accuracy cannot be overstated, as the adequate surgical procedure for each disease is completely different. For instance, in patients with stenosis due to reflux, an antireflux Nissen or Collis-Nissen procedure, with posterior dilation, proves adequate to restore the esophagus. On the contrary, a range of therapeutic options exist. ${ }^{8,11}$ Nevertheless, Heller myotomy procedures accompanied by partial fundoplication allowed for adequate recovery in all our patients. Finally, in patients with congenital stenosis of the esophagus, the presence of embryonic remnants precludes treatment through esophageal dilations. ${ }^{2,3}$ As put forward earlier, this type of patients required a more complex treatment involving resection of the affected segment with end-to-end anastomosis, and one of them presented anastomotic leakage. Of note, all 33 patients in this trial were laparoscopically intervened with endoscopic control, without complications arising from this approach.

\section{Conclusions}

The main takeaways from our study can be summarized as follows:

- Discerning among different etiologies in children with obstruction of the lower third of the esophagus is of utmost importance, given that the appropriate surgical option depends on diagnosis.

- Radiological and endoscopic procedures allow for adequate discrimination between alternative diagnoses.

Once diagnosis has been established, the required surgical procedure can be performed safely by means of minimally invasive techniques.

\section{Ethical Statement}

This protocol was approved by "Jefatura de Enseñanza, Hospital Infantil Privado" and all subjects involved signed an informed consent.

\section{Disclosure Statement}

The authors have no conflict of interest to declare.

\section{Funding Information}

No funding was received for this article.

\section{References}

1. Dohil R, Hassall E. Esophageal stenosis in children. Gastrointest Endosc Clin North Am 1998;8:369-390.

2. Takamizawa S, Tsugawa C, Mouri N, Satoh S, Kanegawa $\mathrm{K}$, Nishijima E, et al. Congenital esophageal stenosis: Therapeutic strategy based on etiology. J Pediatr Surg 2002;37:197-201. 
3. Romeo E, Foschia F, Angelis P, Caldaro T, di Abriola GF, Gambitta R, et al. Endoscopic management of congenital esophageal stenosis. J Pediatr Surg 2011;46:838-841.

4. Nihoul-Fékété C, De Backer A, Lotart-Jacob S, Pellerin D. Congenital esophageal stenosis. A review of 20 cases. Pediatr Surg Int 1987;2:86-92.

5. Zouari M, Kamoun H, Bouthour H, Abdallah RB, Hiel Y, Malek RB, et al. Peptic oesophageal stricture in children: Management problems. Afr J Ped Surg 2014;11:22-25.

6. Numanoglu A, Millar AJW, Brown RA, Rode H. Gastroesophageal reflux strictures in children, management and outcome. Pediatric Surg Int 2005;21:631-634.

7. al-Bassam A. Surgical management of severe peptic esophageal stricture in children. Hepatogastroenterology 2003;50:714-717.

8. Caldaro T, Familiari P, Romeo EF, Gigante G, Marchese M, Contini ACI, et al. Treatment of esophageal achalasia in children: Today and tomorrow. J Pediat Surg 2015;50:726-730.

9. Gilger MA, El-Serag HB, Gold BD, Dietrich CL, Tsou V, McDuffie A, et al. Prevalence of endoscopic findings of erosive esophagitis in children. A population-based study. J Pediatr Gastroenterol Nutr 2008;47:141-146.

10. Mayberry JF, Mayell MJ. Epidemiological study of achalasia in children. Gut 1988;29:90-93.
11. Vaezi MF, Pandolfino JE, Vela MF. ACG clinical guideline: Diagnosis and management of achalasia. Am J Gastroenterol 2013;108:1238-1249.

12. Allgrove J, Clayden GS, Grant DB, Macaulay JC. Familiar glucocorticoid deficiency with achalasia of the cardia and deficient tear production. Lancet 1978;311:1284-1286.

13. Bluestone CD, Kerry R, Sieber WK. Congenital esophageal stenosis. Laryngoscope 1969;79:1095-1104.

14. Achem SR, Devault KR. Dysphagia in aging. J Clin Gastroenterol 2005;39:357-371.

15. Kahrilas PJ, Kishk SM, Helm JF, Dodds WJ, Haring JM, Hogan WJ. Comparison of pseudoachalasia and achalasia. Am J Med 1987;82:439-446.

Address correspondence to: Carlos García-Hernández, MD

Circuito Valle Dorado 12

Col. Loma de Valle Escondido C.P. 52930, Atizapán de Zaragoza Estado de México

México

E-mail: carloscirped@hotmail.com 